\title{
Observer Design for Needle Steering Using Task-Induced Symmetry and Reduction *
}

\author{
Vinutha Kallem* Dong Eui Chang ${ }^{* *}$ Noah J. Cowan ${ }^{* * *}$ \\ * Department of Mechanical Engineering and Applied Mechanics, \\ University of Pennsylvania (e-mail: vkallem@seas.upenn.edu) \\ ** Department of Applied Mathematics, University of Waterloo \\ (e-mail: dechang@math.uwaterloo.ca) \\ *** Department of Mechanical Engineering, Johns Hopkins University \\ (email: ncowan@jhu.upenn.edu)
}

\begin{abstract}
Reduction induced either by the inherent dynamics of a system or control task at hand is often advantageous for analysis and control of the system. In the current work we consider state estimation for flexible, tip-steerable needle insertions. Specifically, we design observers that exploit the symmetry induced by the task of controlling the needle to a desired plane. These needles curve inside the tissue as they are inserted into it and have been modeled as a six degree of freedom nonholonomic system. Several planning and control algorithms have been proposed to automate needle insertions so as to improve their accuracy. Central to these algorithms is the estimation of the needle states (position and orientation of the needle tip) from only the needle-tip position measurements; needle-tip orientation cannot be directly measured since the needle shaft is very thin. We first show that the needle steering system is an observable system and then illustrate how the planar task decomposes the configuration space into a reduced space and a fiber space. Finally, we design an observer on the reduced space and an embedded-space observer on the fiber space.
\end{abstract}

Keywords: Nonholonomic control, Needle steering, Symmetry and Reduction, Observer design, Lie groups

\section{INTRODUCTION}

Needles are pervasive in percutaneous therapies for biopsies, bracytherapy and drug delivery. For these procedures, accurate placement of the needle tip is crucial for the success of the procedure. Needles have to be inserted into tissue accurately to reach a specific target while avoiding bones and sensitive organs. Improving needle targeting accuracy and expanding the applicability of needle interventions in general, involves actively steering a needle as it is being inserted. Physicians often rely on pre- or intraoperative medical imaging to guide a needle to its target. Several factors limit performance, including the amount of steering that a needle affords after it is inserted, noisy sensors, imperfect actuators, and tissue deformations. Furthermore, navigation in 3D under image guidance by manipulating the needle at its base (from outside the patient) requires profound spatial reasoning skills and extensive training.

In the past few years, there has been a large effort to overcome these limitations by improving insertion accuracy through use of robotic systems or needle placement devices for needle insertions [DiMaio et al., 2006, Stoianovici et al., 1998, Wei et al., 2004b], better imaging modalities [Abolhassani and Patel, 2006, Ding et al., 2002, Wei et al., 2004a], needle tissue interaction models [Glozman

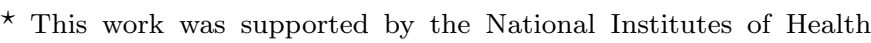
under grants R21-EB00345 and R01- EB006435.
}

and Shoham, 2004, Webster et al., 2006], and planning algorithms [Alterovitz et al., 2005, 2007, Park et al., 2005, DiMaio and Salcudean, 2003, Duindam et al., 2008]. Concurrently, new needle design research, such as on curved or pre-bent needles, flexible needles, needles with a stylus at the tip and flexible steerable-tip needles, has aimed to expand the applicability of needle insertions in medical procedures by their ability to reach locations in the tissue that cannot be reached by traditional rigid symmetric-tip needles [Okazawa et al., 2005, Webster et al., 2005, DiMaio and Salcudean, 2003]. We refer to [Abolhassani et al., 2007, Reed et al., 2010, Cowan et al., 2011] for recent surveys on needle insertions, modeling and simulations.

In the present work we consider flexible, tip-steerable needle insertions into tissue. When inserted into tissue, these steerable needles follow a circular path due to asymmetric cutting forces at the needle tip. Webster et al. [2006] model this effect using a nonholonomic kinematic system. This system has six states and two inputs, namely the forward insertion and rotation of the needle at the base. Alterovitz et al. [2005, 2007] develop planners that rely on the needle staying within a specified $2 \mathrm{D}$ plane, and construct a sequence of circular arcs to reach the target while avoiding obstacles. Kallem and Cowan [2007, 2009] design and implement a controller that steers a needle to stay within a desired 2D plane. Duindam et al. [2008] propose a 3D motion planning algorithm for flexible steerable-tip needle insertions with spherical obstacles in 
the workspace. All these control and planning algorithms assume that the whole needle state can be measured or estimated from the measured states. In an operating room image-guided needle insertions are performed using ultrasound, CT scans, MR imaging, magnetic tracking or a combination of them. Since the needle is thin, there is no direct method to measure the orientation of the bevel using the stereo cameras. To our knowledge, the needle orientation cannot yet be measured using any other imaging modality, be it ultrasound, X-ray, fluoroscopy or MRI. However, this rotation information is necessary for control and planning algorithms. Further, segmenting or tracking the needle in these images is non-trivial as these images are very noisy. Hence, designing robust modelbased observers which use noisy tip position measurements to estimate all the needle states is crucial.

Traditionally, pose estimation from images such as in the structure from motion (SFM) literature is performed based on sequential video images of a moving cloud of points; this observation typically over-constrains the underlying rigid transformation. The problem is conceived as a nonlinear optimization problem, where the objective is to find the best-fit rigid body transformation to the image data collected in two views, or in an image sequence [Arun et al., 1987, Umeyama, 1991, Soatto et al., 1996, Tomasi and Kanade, 1992, Vidal et al., 2004, Vidal and Hartley, 2004]. When the dynamical model of a system is known and only a part of the rigid body transformation can be directly measured (as in the needle steering system), the problem becomes one of designing an observer that converges - asymptotically or in finite time - to the actual transformation. Here we show that the rotation of the needle tip may be inferred from the measurements of the needle tip position over time, and we then present dead-beat and asymptotic observers that exploit the taskinduced reduction to estimate the full needle pose.

In this paper, we present an asymptotic observer that uses the task-induced reduction developed in [Kallem and Cowan, 2007, 2009]. In Section 2, we review the kinematics of flexible, steerable-tip needle insertions. We then show the observability of the needle steering system when only the position of the needle tip can be measured (Section 3). In Section 4, we discuss the notion of "taskinduced" symmetry reduction and review it in the context of planar control of needle steering. Using the task-induced reduction, we first develop an observer to estimate the $x$ position, the pitch of the needle tip $(\beta)$ and the roll of the needle $(\gamma)$ from just $x$ position measurements (Section 5.2). In Section 5.3 we develop an observer for the other three states $(y, z$ and yaw $(\alpha))$ by embedding $\mathrm{SE}(2)$ into a higher dimensional manifold, $\mathbb{R}^{4}$. We then present successful illustrations of the observer in Section 6 .

\section{FLEXIBLE STEERABLE-TIP NEEDLE KINEMATICS}

As the flexible bevel-tip needle (Figure 1(a)) is inserted, the asymmetry of the bevel creates a moment at the needle tip, deflecting the needle and causing it to follow a circular arc. As the needle base is rotated, the bevel tip is reoriented in space, so that subsequent insertion follows another circular arc in 3D space. If the rotation amount is

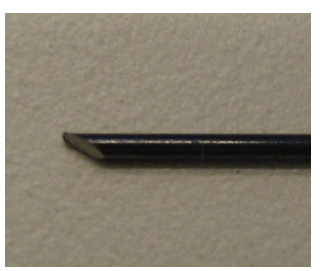

(a)

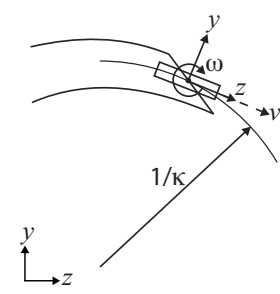

(b)
Fig. 1. (a) Flexible, steerable-tip needle. (b) Kinematic generalized unicycle model of needle steering. The curvature of the path the needle follows inside the tissue is $\kappa$. This figure is reproduced from Webster et al. [2006] with permission from the authors.

not a multiple of $180^{\circ}$ then the new circular arc lies in a different plane from the first one. A sequence of these arcs can be used to reach a point in the tissue while avoiding sensitive organs and bones.

Webster et al. [2006] develop a nonholonomic model of flexible, steerable-tip needle steering; we briefly review the model here for completeness. The model consists of six states, namely the orientation and position of the needle tip, and two inputs which are the insertion and rotation speeds. The needle steering model (see Figure 1(b)) is a generalization of the nonholonomic unicycle model, and neglects torsional compliance of the needle shaft. The state of the system lies on the Special Euclidean manifold, $\mathrm{SE}(3)$. Let $v$ denote the insertion speed and $\omega$ the rotation speed of the needle. The kinematic model of needle steering is given by

$$
\left(g^{-1} \dot{g}\right)^{\vee}=\mathbf{V}_{1} v+\mathbf{V}_{2} \omega,
$$

where $g=\left[\begin{array}{cc}R & \boldsymbol{p} \\ 0^{T} & 1\end{array}\right] \in \mathrm{SE}(3)$ with $R \in \mathrm{SO}(3)$ representing the orientation of the needle and $\boldsymbol{p} \in \mathbb{R}^{3}$ the position of the needle tip. ${ }^{\vee}$ and ${ }^{\wedge}$ denote the usual isomorphism between $\mathfrak{s e}(3)$ and $\mathbb{R}^{6}$. The control vector fields are given by $\mathbf{V}_{1}=\left[\begin{array}{llllll}0 & 0 & 1 & \kappa & 0 & 0\end{array}\right]^{T}$ and $\mathbf{V}_{2}=\left[\begin{array}{llllll}0 & 0 & 0 & 0 & 0 & 1\end{array}\right]^{T}$ and $\kappa$ is the radius of curvature of the path the needle will follow inside the tissue as it is being inserted. The vector field $\mathbf{V}_{1}$ corresponds to the insertion of the needle into the tissue and $\mathbf{V}_{2}$ corresponds to the rotation of the needle outside the tissue (or human). This nonholonomic system has a degree of nonholonomy of four. Note that this model is only valid for forward insertions of the needle into the tissue; during the removal of the needle from the tissue, there are no cutting forces on the needle tip and hence the needle follows the path (in reverse) it followed during the forward insertion into the tissue.

\section{ESTIMATION OF 6-DOF NEEDLE POSE FROM POSITION MEASUREMENTS}

Assume that we have a needle tip position over time, denoted by $\boldsymbol{p}(t)$. Here on we assume also that the forward insertion of the needle is held constant at $v(t)=v$. This assumption is equivalent to parameterizing the system based on insertion distance rather than time. Let $\boldsymbol{e}_{i}^{\prime} s$ for $i=1,2,3$ denote the principal unit vectors in three dimensions. Two successive time derivatives of $\boldsymbol{p}(t)$ and using the kinematic model (1) yield 
and

$$
\dot{\boldsymbol{p}}(t)=R(t) \boldsymbol{e}_{3} v
$$

$\ddot{\boldsymbol{p}}(t)=-\kappa R(t) \boldsymbol{e}_{2} v$

and their cross product is given by

$$
\dot{\boldsymbol{p}}(t) \times \ddot{\boldsymbol{p}}(t)=\kappa R(t) \boldsymbol{e}_{1} v^{2} .
$$

Provided that $v>0$, the rotation of the needle tip, $R(t)$, can be determined:

$$
R(t)=\frac{1}{v}\left[\frac{1}{k v} \dot{\boldsymbol{p}}(t) \times \ddot{\boldsymbol{p}}(t)-\frac{1}{k} \ddot{\boldsymbol{p}}(t) \dot{\boldsymbol{p}}(t)\right] .
$$

This analysis shows that the orientation is indeed observable from position measurements if $v>0$.

Equation (2) can directly be used as an observer, but in practice this would imply finite difference approximations to the first and second time derivatives of the needle tip position because of the availability of only discrete samples of $\boldsymbol{p}(t)$. Also, since the needle steering system is nonlinear, control and observer designs are inherently coupled and they need to be developed simultaneously. Here we consider the task of controlling the flexible, steerable-tip needle to a desired 2D plane and we use the controller developed in [Kallem and Cowan, 2007, 2009]. The motivation on this task is to use it in conjunction with the 2D planners developed by [Alterovitz et al., 2005,2007 to drive the needle to a desired goal while avoiding obstacles. We first decompose the configuration space into a reduced space (where the controller acts) and a fiber space (where the planner acts) using "task-induced" reduction and use it further to design observers for needle steering.

\section{TASK-INDUCED SYMMETRY AND REDUCTION}

Reduction induced either by the inherent dynamics of the system or the control task at hand is often advantageous for analysis and control of the system [Bloch, 2003, Marsden et al., 1990]. Here we use "task-induced" reduction [Kallem et al., 2007, 2010] to design observers for needle steering. The "task-induced" reduction is extrinsic to the system and the symmetry comes from the control task in contrast with the Lagrangian symmetry and reduction.

Proposition [Kallem et al., 2007, 2010] Let $G$ be a Lie group and $\mathfrak{g}$ its Lie algebra. Consider the left-invariant kinematic system

$$
g^{-1} \dot{g}=\xi_{0}+\sum_{i=1}^{k} \xi_{i} u_{i}
$$

where $g \in G, \xi_{i} \in \mathfrak{g}$, and $u_{i}$ 's for $i=1,2, \ldots, k$ are inputs to the system. Let the control task be defined as the zero value of a functional $\phi: G \rightarrow \mathbb{R}$. Let $H$ be the subgroup of $G$ invariant to the task, namely $H=\{h \in G: \forall g \in$ $G, \phi(h g)=\phi(g)\}$. Since $H$ is a subgroup of $G$ there exists a bundle projection $\pi: G \rightarrow B=G / H$. The vector field on the reduced space, $B$, can be calculated as

$$
\dot{r}=f_{0}(r)+\sum_{i=1}^{k} f_{i}(r) u_{i}
$$

where $f_{i}(r)=T_{g} \pi \cdot g \xi_{i}$, and the fiber dynamics are given by

$$
h^{-1} \dot{h}=\operatorname{Ad}_{s(r)}\left(\xi_{0}+\sum_{i=1}^{k} \xi_{i} u_{i}-s(r)^{-1} T s \cdot \dot{r}\right) .
$$

Since the fiber space, $H$, does not affect the control task, we need to consider the state evolution only in the base space for achieving the control task.

The needle steering system is a left-invariant system with $G=\mathrm{SE}(3)$ and the control task of driving the needle to the plane is invariant to rigid body transformations in the $y$ - $z$ plane making $H=\mathrm{SE}(2)$. The reduced system is the base space of the principal fiber bundle $\left(\mathrm{SE}(3), \pi, S^{2} \times \mathbb{R}\right)$. The projection map of the bundle is given by

$$
\pi(g)=\pi(R, \boldsymbol{p})=\left(R^{-1} e_{1}, \boldsymbol{p}^{T} e_{1}\right)
$$

where $g=(R, \boldsymbol{p}) \in \mathrm{SE}(3)$. Note that the reduced space is $S^{2} \times \mathbb{R}$ and the fiber space is $\mathrm{SE}(2)$. Kallem and Cowan [2007, 2009] use Z-Y-X fixed angles as generalized coordinates to parameterize $R$, the needle-tip orientation. Let $\gamma$ be the roll of the needle, $\beta$ be the pitch of the needle out of the plane and $\alpha$ be the yaw of the needle in the plane. Let the position of the needle-tip be $\boldsymbol{p}=$ $\left[\begin{array}{lll}x & y & z\end{array}\right]^{T} \in \mathbb{R}^{3}$ which can be measured by imaging system. Since these local coordinates are well defined on $\mathcal{U}=$ $\left\{[x y z \alpha \beta \gamma]^{T} \in \mathbb{R}^{6}: \alpha, \gamma \in \mathbb{R} \bmod 2 \pi, \beta \in(-\pi / 2, \pi / 2)\right\}$, the planar controller and the observer are local; we observe in the simulations and experiments that the domain of attraction is quite large (see Section 6).

Here on, we assume that $v=1$ as this assumption is a equivalent to parameterizing the system based on insertion distance rather than time. Let $\boldsymbol{r}=\left[\begin{array}{lll}r_{1} & r_{2} & r_{3}\end{array}\right]^{T}=$ $\left[\begin{array}{lll}x & \beta & \gamma\end{array}\right]^{T}$ denote the state vector of the "reduced" order system which is given by

$$
\begin{aligned}
\dot{\boldsymbol{r}} & =\left[\begin{array}{c}
\sin r_{2} \\
\kappa \sin r_{3} \\
-\kappa \cos r_{3} \tan r_{2}
\end{array}\right]+\left[\begin{array}{l}
0 \\
0 \\
1
\end{array}\right] \omega \\
y_{r} & =r_{1},
\end{aligned}
$$

where $y_{r}$ is the measured state of the reduced system. The fiber space state $\boldsymbol{h}=\left[\begin{array}{lll}h_{1} & h_{2} & h_{3}\end{array}\right]^{T}=\left[\begin{array}{lll}y & z & \alpha\end{array}\right]^{T}$ evolves as

$$
\begin{aligned}
\dot{h} & =\left[\begin{array}{c}
-\sin h_{3} \cos r_{2} \\
\cos h_{3} \cos r_{2} \\
\kappa \sec r_{2} \cos r_{3}
\end{array}\right] \\
\boldsymbol{y}_{h} & =\left[\begin{array}{c}
h_{1} \\
h_{2}
\end{array}\right] .
\end{aligned}
$$

Note that $\boldsymbol{r}=0$ corresponds to stabilizing the needle to the $y$ - $z$ plane.

\section{ASYMPTOTIC OBSERVER USING REDUCTION AND STATE IMMERSION}

\subsection{Previous work on state immersion}

Linearizing nonlinear systems has tremendous advantages in systems analysis and in designing controllers and observers. Jacobian linearization is a first order Taylor series approximation of a nonlinear system near an equilibrium point and is useful in analyzing stability properties. In feedback linearization an exact change of coordinates of the state space variables (both the states and inputs) is obtained by taking successive Lie derivatives of the output to result in a global change of coordinates under certain conditions and sometimes this may only result in a local (perhaps large) change of coordinates Khalil [2002]. In Carleman linearization the configuration space is immersed in a higher dimensional space to obtain a 
linear system. In Carleman linearization, an infinite Taylor series expansion is performed on the system around an equilibrium point, and all the monomials of the states variables are assigned as the new state variables. This technique results in a bilinear system that has an infinite number of states in general. The Taylor expansion in the Carleman linearization can be truncated to obtain a finite order bilinear system, but this makes the linearization approximate. By increasing the order of the Taylor expansion, better approximations can be obtained. More details can be found in [Kowalski and Steeb, 1991, Sastry, 1999].

Below, we present an asymptotic observer for the reduced space using a change of coordinates obtained through feedback linearization. Then we use a linear model to represent three-state fiber dynamics by state immersion into a finite higher dimensional manifold. We use the output and its derivatives in a similar manner as that in feedback linearization, and embed the $\mathrm{SE}(2)$ manifold into $\mathbb{R}^{4}$ to obtain a linear system and design an observer for the fiber space states. It is important to note that our embedding for $\mathrm{SE}(2)$ in higher dimensional space is exact unlike Carlemen linearization (see Section 5.3).

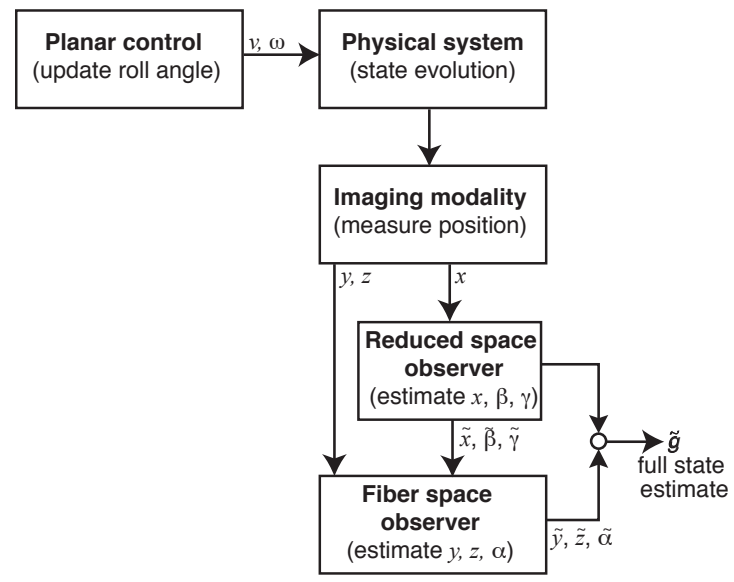

Fig. 2. Coupled reduced space and fiber space asymptotic observers. The reduced space observer estimates $x$ position, pitch and roll of the needle tip from (noisy) $x$ measurements. The fiber space observer uses these estimates along with the (noisy) $y$ and $z$ measurements and estimates the rest of the states of the needle.

\subsection{Reduced space observer}

The reduced space system can be feedback linearized via a transformation of state and input coordinates [Kallem and Cowan, 2007, 2009]:

$$
\begin{aligned}
& \boldsymbol{s}=\left[r_{1}, \sin r_{2}, \kappa \cos r_{2} \sin r_{3}\right]^{T}, \\
& u=-\kappa^{2} \sin r_{2}+\kappa \cos r_{2} \cos r_{3} \omega .
\end{aligned}
$$

The state equations in the feedback linearized form are:

$$
\begin{aligned}
& \dot{\boldsymbol{s}}=A_{r} \boldsymbol{s}+B_{r} u=\left[\begin{array}{lll}
0 & 1 & 0 \\
0 & 0 & 1 \\
0 & 0 & 0
\end{array}\right] \boldsymbol{s}+\left[\begin{array}{l}
0 \\
0 \\
1
\end{array}\right] u \\
& y_{r}=C_{r} \boldsymbol{s}=\left[\begin{array}{lll}
1 & 0 & 0
\end{array}\right] \boldsymbol{s} .
\end{aligned}
$$

A full state Luenberger observer with the following dynamics estimates all the reduced states from the output:

$$
\begin{aligned}
\dot{\tilde{\boldsymbol{s}}} & =A_{r} \tilde{\boldsymbol{s}}+B_{r} u+L_{r}\left(y_{r}-\tilde{y}_{r}\right) \\
\tilde{y}_{r} & =C_{r} \tilde{\boldsymbol{s}} .
\end{aligned}
$$

\subsection{Fiber space observer though state immersion}

Assume that the needle tip is driven to the desired plane (the $y-z$ plane). At this configuration, the needle tip position and orientation is such that $x=0, \beta=0$ and $\gamma=0$ or $\gamma=180^{\circ}$. For the current analysis, let us assume that $\gamma=0$. The other three states lie on the $\mathrm{SE}(2)$ manifold and evolve with the following dynamics:

$$
\dot{\boldsymbol{h}}=\left[\begin{array}{c}
-\sin \alpha \\
\cos \alpha \\
\kappa
\end{array}\right] \text {. }
$$

Consider the embedding, $\varphi: \operatorname{SE}(2) \rightarrow \mathbb{R}^{4}$ defined by

$$
\varphi(\boldsymbol{h})=\left[\begin{array}{c}
y \\
z \\
-\sin \alpha \\
\cos \alpha
\end{array}\right]
$$

Note that the Jacobian, $\frac{\partial \varphi}{\partial h}$, is full rank everywhere. Also $\varphi$ is a smooth mapping and there is a one-to-one mapping between $\mathrm{SE}(2)$ and $\varphi(\operatorname{SE}(2))$. In these coordinates, $\hbar \in$ $\varphi(\mathrm{SE}(2)) \subset \mathbb{R}^{4}$, the fiber dynamics are

$$
\dot{\hbar}=A_{h} \hbar=\left[\begin{array}{cccc}
0 & 0 & 1 & 0 \\
0 & 0 & 0 & 1 \\
0 & 0 & 0 & -\kappa \\
0 & 0 & \kappa & 0
\end{array}\right] \hbar \text {. }
$$

Thus, by embedding $\mathrm{SE}(2)$ into a higher dimensional $\mathbb{R}^{4}$, we obtain a linear system. Also, since we can measure the needle tip position, the output states of this system are given by

$$
\boldsymbol{y}_{h}=C_{h} \hbar=\left[\begin{array}{llll}
1 & 0 & 0 & 0 \\
0 & 1 & 0 & 0
\end{array}\right] \hbar .
$$

The system $\left(A_{h}, C_{h}\right)$ is observable and the following Luenberger observer will estimate the the state vector $\hbar$ :

$$
\begin{aligned}
\dot{\tilde{\hbar}} & =A_{h} \hbar+L_{h}\left(\boldsymbol{y}_{h}-\tilde{\boldsymbol{y}}_{h}\right) \\
\tilde{\boldsymbol{y}}_{h} & =C_{h} \tilde{\hbar},
\end{aligned}
$$

where $\left(A_{h}-L_{h} C_{h}\right)$ is Hurwitz. Note that in general $\tilde{h}(t)$ does not lie on $\varphi(\operatorname{SE}(2))$, but as $t \rightarrow \infty$, it lies on $\varphi(\operatorname{SE}(2))$. The estimate of fiber space state, $\tilde{h}$, is given by

$$
\tilde{h}=\left[\begin{array}{c}
\tilde{\hbar}_{1} \\
\tilde{\hbar}_{2} \\
\arctan 2\left(-\tilde{\hbar}_{3}, \tilde{\hbar}_{4}\right)
\end{array}\right] .
$$

\subsection{Coupled reduced space and fiber space observers}

Now, when the needle is being driven towards the desired plane ( $y-z$ plane), the reduced space observer can feed into the fiber space observer to estimate the full 6-DOF pose of the needle from just position measurements; see Figure 2. In this case, the fiber space dynamics is given by (4) and the fiber space observer is given by

$$
\begin{aligned}
\dot{\tilde{\hbar}} & =A_{h} T \hbar+L_{h}\left(\boldsymbol{y}_{h}-\tilde{\boldsymbol{y}}_{h}\right) \\
\tilde{\boldsymbol{y}}_{h} & =C_{h} \tilde{\hbar},
\end{aligned}
$$

where

$$
T=\left[\begin{array}{cccc}
\cos \tilde{\beta} & 0 & 0 & 0 \\
0 & \cos \tilde{\beta} & 0 & 0 \\
0 & 0 & \cos \tilde{\gamma} \sec \tilde{\beta} & 0 \\
0 & 0 & 0 & \cos \tilde{\gamma} \sec \tilde{\beta}
\end{array}\right]
$$



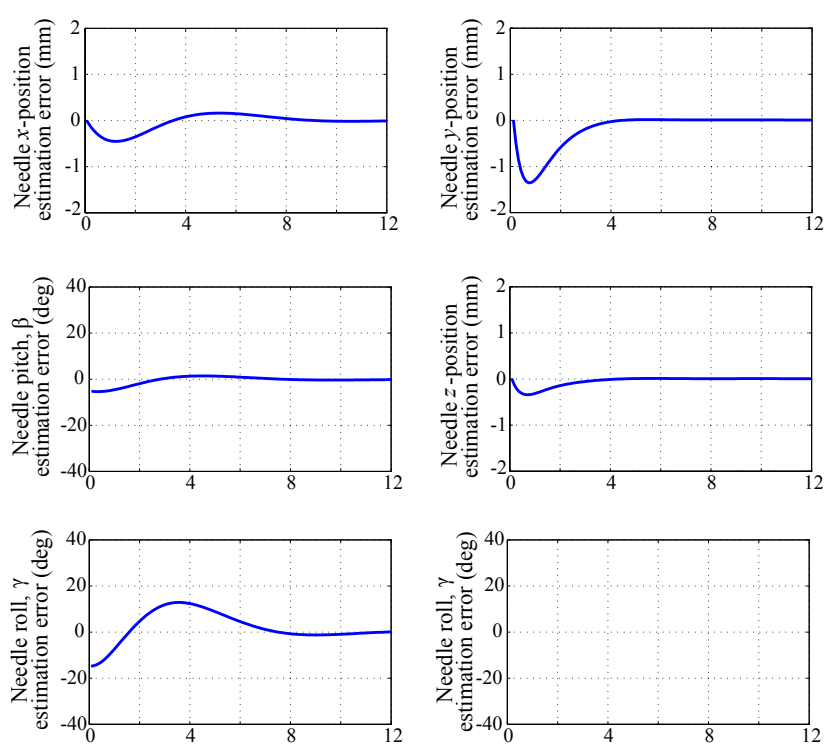

Needle Insertion Distance (cm)

Fig. 3. Asymptotic observer to estimate the full 6-DOF pose of needle steering from just the position measurements in MATLAB simulations. The radius of curvature of the needle in the tissue is taken as $12.2 \mathrm{~cm}$, tip position data is collected every $1 \mathrm{~mm}$ of insertion with no measurement noise. The estimation errors converge to zero in all the six degrees of freedom.

Note that when the needle is stabilized to the desired plane $\left(x=0, \beta=0^{\circ}\right.$ and $\left.\gamma=0^{\circ}\right), T=I$ and the observer converges to the one given in (13).

\section{SIMULATION RESULTS}

We present MATLAB simulations to illustrate the working of the proposed observer. The radius of curvature is assumed to be $12.2 \mathrm{~cm}$. Figure 3 is one such simulation with no noise in the position measurements. Needle position data was collected every $1 \mathrm{~mm}$ of insertion. The figure shows the estimation errors in all the six degrees of freedom. The left column shows the estimation errors of the reduced space variables and the right column shows estimation errors in the fiber variables. The estimation error decreases from an initial error and goes to zero asymptotically. Figure 4 is an example simulation run, in which the measured position data is noisy. Here, noisy needle tip position data was collected every $1 \mathrm{~mm}$ of insertion, with measurement noise that is uniformly distributed within $\pm 1 \mathrm{~mm}$ in each of three coordinates of the position measurement. In this example, we can see that that the estimate errors decrease but oscillate around zero due to the presence of noise. It should be noted that high gains are used in the planar controller and observer to obtain fast convergence of the needle to the $y$ - $z$ plane. If less aggressive gains are used, then the steady state oscillations are lower, thus reducing the steady state variance in estimation. We have also successfully used this observer in implementations of control and planning algorithms implemented on phantom tissue [Reed et al., 2008, 2010]; although the present paper is the first to report on the observer design itself, which has not been been previously reported.
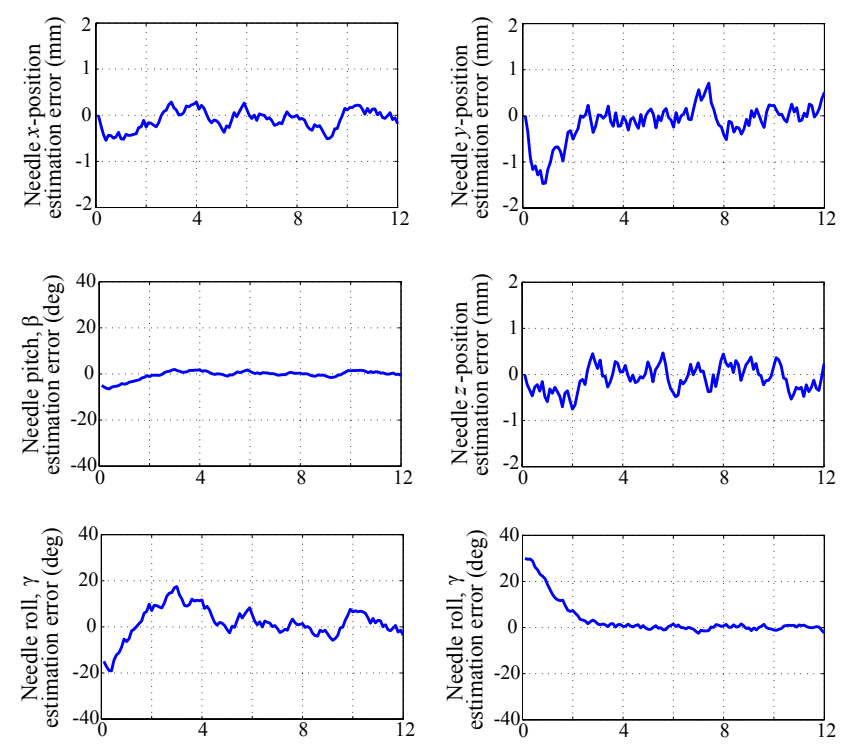

Needle Insertion Distance $(\mathrm{cm})$

Fig. 4. Asymptotic observer to estimate the full 6-DOF pose of needle steering from just the position measurements in MATLAB simulations. The radius of curvature of the needle in the tissue is taken as $12.2 \mathrm{~cm}$. Noisy needle tip position data is collected every $1 \mathrm{~mm}$ of insertion, with measurement noise that is uniformly distributed within $\pm 1 \mathrm{~mm}$. The estimation errors converge to zero in all the six degrees of freedom with a low steady-state variance.

\section{CONCLUSIONS}

In this work, we develop an observer on the group of rigid-body transformations for needle steering using "taskinduced" symmetry and reduction with only the needle tip position measurements. We consider the case of stabilizing the needle tip to a desired $2 \mathrm{D}$ plane and decompose the space into a reduced space and a fiber space. For the reduced space observer, we convert the system into a linear system by a change of coordinates and for the fiber space we embed the space into a higher dimensional space to obtain a linear system. We implement the observer successfully in MATLAB simulations and show that this observer can be used for planning and control purposes in laboratory experiments performed on phantom tissue [Reed et al., 2008, 2010]. This observer uses local coordinates making the domain of attraction of the observer local too. Future work to design observer on rigid-body transformations that does not require local coordinates would be very useful.

\section{REFERENCES}

Niki Abolhassani and Rajini V. Patel. Deflection of a flexible needle during needle insertion into soft tissue. In Conf. of IEEE EMBS, pages 3858-3861, August 2006.

Niki Abolhassani, Rajni Patel, and Mehrdad Moallem. Needle insertion into soft tissue: a survey. Med. Engineering and Physics, 29:413-431, 2007.

Ron Alterovitz, Andrew Lim, Ken Goldberg, Gregory S. Chirikjian, and Allison M Okamura. Steering flexible needles under markov motion uncertainty. In Proc. 
IEEE/RSJ Int. Conf. Intell. Robots Syst., pages 1570 1575. IEEE/RSJ, August 2005.

Ron Alterovitz, Thierry Simeon, , and Ken Goldberg. The stochastic motion roadmap: A sampling framework for planning with markov motion uncertainty. In Robotics: Science and Systems, June 2007.

K S Arun, T S Huang, and S D Blostein. Least-squares fitting of two 3-D point sets. IEEE Trans. Pattern Anal. Mach. Intell., 9:698-700, 1987.

A. Bloch. Nonholonomic Mechanics and Control, volume 24. Springer, NY, 2003.

Noah J Cowan, Allison M Okamura, Ken Goldberg, Gregory S Chirikjian, Ron Alterovitz, Kyle B Reed, Vinutha Kallem, Gabor Fichtinger, Wooram Park, and Sarthak Misra. Robotic needle steering: Design, modeling, planning, and image guidance. In Surgical Robotics-Systems, Applications, and Visions. Springer, 2011.

S P DiMaio, G S Fischer, S J Haker, N Hata, I Iordachita, C M Tempany, R Kikinis, and G Fichtinger. A system for MRI-guided prostate interventions. In Proc. Int. Conf. Biomedical Robotics and Biomechatronics, pages 68-73, 2006.

Simon P DiMaio and Septimiu E Salcudean. Needle insertion modeling and simulation. IEEE Trans. Robot. Autom., 19:864-875, 2003.

Mingyue Ding, H. Neale Cardinal, Weiguang Guan, and Aaron Fenster. Automatic needle segmentation in 3D ultrasound images. In SPIE Med. Imaging, 2002.

Vincent Duindam, Ron Alterovitz, Shankar Sastry, and Ken Goldberg. Screw-based motion planning for beveltip flexible needles in 3D environments with obstacles. In Proc. IEEE Int. Conf. Robot. Autom., May 2008.

Daniel Glozman and Moshe Shoham. Flexible needle steering and optimal trajectory planning for percutaneous therapies. In Proc. Med. Image Computing and Computer-Assisted Intervention, pages 137-144, 2004.

Vinutha Kallem and Noah J Cowan. Image-guided control of flexible bevel-tip needles. In Proc. IEEE Int. Conf. Robot. Autom., pages 3015-3020, Rome, Italy, April 2007.

Vinutha Kallem and Noah J. Cowan. Image guidance of flexible tip-steerable needles. IEEE Trans. Robot., 25: 191-196, 2009. doi: 10.1109/TRO.2008.2010357.

Vinutha Kallem, Dong Eui Chang, and Noah J. Cowan. Task-induced symmetry and reduction in kinematic systems with application to needle steering. In Proc. IEEE/RSJ Int. Conf. Intell. Robots Syst., pages 33023308, San Diego, CA, USA, October 2007.

Vinutha Kallem, Dong Eui Chang, and Noah J Cowan. Task-induced symmetry and reduction with application to needle steering. IEEE Trans. Autom. Control, 55: 664-673, 2010.

Hassan K. Khalil. Nonlinear Systems. Prentice Hall, 2 edition, 2002.

Krzysztof Kowalski and Willi-Hans Steeb. Nonlinear Dynamical Systems and Carleman Linearization. World Scientific Publishing Company, 1991.

JE Marsden, R Montgomery, and TS Ratiu. Reduction, Symmetry and Phases in Mechanics. American Mathematical Society, 1990.

Stephen Okazawa, Richelle Ebrahimi, Jason Chuang, Septimiu E Salcudean, and Robert Rohling. Hand-held steerable needle device. IEEE/ASME Trans. Mech., 10
(3):285-296, 2005 .

Wooram Park, Jin Seob Kim, Yu Zhou, Noah J. Cowan, Allison M. Okamura, and Gregory S. Chirikjian. Diffusion-based motion planning for a nonholonomic flexible needle model. In Proc. IEEE Int. Conf. Robot. Autom., pages 4600-4605, Barcelona, Spain, April 2005.

Kyle B. Reed, Vinutha Kallem, Ron Alterovitz, Ken Goldberg, Allison M. Okamura, and Noah J. Cowan. Integrated planning and image-guided control for planar needle-steering. In Proc. IEEE Conf. Biomed. Robot. and Biomechatronics, pages 819-824, Scottsdale, AR, USA, October 2008.

Kyle B Reed, Ann Majewicz, Vinutha Kallem, Ron Alterovitz, Ken Goldberg, Noah J Cowan, and Allison M Okamura. Robot-assisted needle steering. IEEE Robot. Autom. Mag., 2010. In press.

Shankar Sastry. Nonlinear Systems. Springer, 1999.

Stefano Soatto, Roggero Frezza, and Pietro Perona. Motion estimation via dynamic vision. IEEE Trans. Autom. Control, 41:393-413, 1996.

Dan Stoianovici, Louis L. Whitcomb, James H. Anderson, Russell H. Taylor, and Louis R. Kavoussi. A modular surgical robotic system for image guided percutaneous procedures. In Proc. Med. Image Computing and Computer-Assisted Intervention, volume 1496, pages 404-410, October 1998.

Carlo Tomasi and Takeo Kanade. Shape and motion from from imgae streams under orthography: a factorization method. Int. J. Computer Vision, 9:137-154, 1992.

Shinji Umeyama. Least-squares estimation of transformation parameters between two point patterns. IEEE Transactions on Pattern Analysis and Machine Intelligence, 13:376-380, 1991.

Rene Vidal and Richard Hartley. Motion segmentation with missing data using powerfactorization and gpca. In Proc. Computer Vision and Pattern Recognition, volume 2, pages 310-316, 2004.

Rene Vidal, Yi Ma, Stefano Soatto, and Shankar Sastry. Two-view multi body structure from motion. Int. J. Computer Vision, 68:7-25, June 2004.

Robert J. Webster, Jasenka Memisevic, and Allison M. Okamura. Design considerations for robotic needle steering. In Proc. IEEE Int. Conf. Robot. Autom., pages 3588-3594, April 2005.

Robert J. Webster, Jin S. Kim, Noah J. Cowan, Greg S. Chirikjian, and Allison M. Okamura. Nonholonomic modeling of needle steering. Int. J. Robot. Res., 25(5/6): 509-526, May 2006.

Zhouping Wei, L. Gardi, D.B. Downey, and A. Fenster. Oblique needle segmentation for 3D TRUS-guided robot-aided transperineal prostate brachytherapy. In IEEE Biomedical Imaging: Nano to Macro, volume 1, pages 960-963, April 2004a.

Zhouping Wei, Gang Wan, Lori Gardi, Gregory Mills, Donal Downey, and Aaron Fenster. Robot-assisted 3DTRUS guided prostate brachytherapy: system integration and validation. Med. Phys., 31 (3):539-48, March 2004b. 